DISCS, OUTFLOWS, JETS AND HH OBJECTS 


\title{
DISKS AND OUTFLOWS
}

\author{
Luis F. Rodríguez \\ Instituto de Astronomía, UNAM \\ Apdo. Postal 70-264 \\ 04510 México, D. F., MEXICO
}

\begin{abstract}
This paper reviews recent developments in the study of bipolar outflows and their possible collimation by disklike structures. The exciting stars of most bipolar outflows are embedded in dense cores, self-gravitating structures with masses in the range of 1 to $10^{3} M_{\odot}$. In many cases, these dense cores are flattened, with its major axis aligned perpendicular to the axis of the outflow. These flattened structures, the interstellar toroids, appear to play a role in the large scale $(0.1 \mathrm{pc})$ collimation of the bipolar outflows. However, a considerable number of observations point to the presence of collimation on much smaller scales. In particular, the results of Sargent and collaborators have revealed the existence of circumstellar disks (with radii of $\sim 1000 \mathrm{AU}$ ) that are gravitationally bound to their stars. I also review recent models of accretion disks around $T$ Tauri stars have been successful in explaining the general features of the continuum spectra of $T$ Tauri stars from the ultraviolet to the infrared. Some radio results that have not been considered by these models are discussed.

A discussion of radio continuum observations of Herbig-Haro objects and their exciting sources is also given. In particular, it is emphasized that the Herbig-Haro objects associated with massive stars are more luminous in the radio and exhibit larger line widths in the optical than the classic $\mathrm{HH}$ objects (that is, those associated with low-luminosity, young stars).
\end{abstract}

\section{INTRODUCTION}

Perhaps the most remarkable property of the molecular outflows is that they frequently exhibit a bipolar geometry. A natural explanation for this characteristic is that the young stars undergoing the outflow phase are surrounded by disks, toroids, or in general some kind of flattened gaseous structures that could provide the observed collimation. The first part of this paper reviews some recent observational and theoretical contributions to our understanding of the disk-outflow connection. Considerable advances have been made, and it seems likely that in the near future observers and theoreticians will even get to be talking of the same thing.

In the second part of this paper I review a field that has experimented rapid growth in the last couple of years: the observations of radio continuum emission from Herbig-Haro objects and their exciting sources. After the detection of $\mathrm{HH} 1$ and $\mathrm{HH} 2$ and their exciting source VLA 1 (Pravdo et al. 1985), searches for radio continuum emission toward other classic HH objects (by classic HH objects I mean those excited by a low-mass, low-luminosity young star) proved unsuccessful (Lane 1989; Curiel 
et al. 1989). However, it has been known for many years (Gull et al. 1973; Münch 1977; Gyulbudaghian, Glushkov, and Denisyuk 1978; Cantó et al. 1980; Meaburn and White 1982; and Strom et al. 1986) that the HH objects can also be found in association with more massive stars. These $\mathrm{HH}$ objects associated with massive stars have turned out to be intrinsically much brighter than their smaller brothers and they, as well as their exciting sources, can be detected and studied in the radio continuum.

\section{DENSE GAS AROUND STARS THAT POWER OUTFLOWS}

I will discuss the topic of the presence of gaseous structures around young stars that are powering bipolar outflows starting from what appears to be more firmly established and moving to progressively more speculative realms. In general, it is known that the exciting stars of outflows are, in most cases, embedded in dense cores (Torrelles et al. 1983; 1986; Myers et al. 1988; Anglada et al. 1989). These are objects that typically have dimensions of a few tenths of a parsec, masses from 1 to $10^{3}$ solar masses, and molecular hydrogen densities exceeding a few times $10^{3}$ $\mathrm{cm}^{-3}$ (Myers 1985).

The dense cores are better studied in the line emission of molecules like ammonia $\left(\mathrm{NH}_{3}\right)$ and carbon monosulphide (CS), which are tracers of dense gas. The association of stars with dense cores has two interesting implications. First, the dense cores are usually as or more massive than the embedded star. It then seems reasonable to conclude that the star condensed from gas that was originally in the core. Second, the stars usually appear projected very close $(\leq 0.1 \mathrm{pc})$ to the peak of the core. Since drift velocities of $\sim 1 \mathrm{~km} \mathrm{~s}^{-1}$ between the star and the core are not unreasonable, one reaches the remarkable conclusion that the embedded star is younger that $10^{5}$ years.

In many of the sources studied the dense core exhibits a flattened geometry, with its major axis aligned perpendicular to the outflow axis. Torrelles et al. (1983) refer to these flattened cores as interstellar toroids, interstellar because they have large, interstellar dimensions, and toroids because they are usually fairly thick structures and the term disk, favored by many authors, seems more adequate for thinner structures. A recent example of an interstellar, flattened structure aligned perpendicular to the axis of a bipolar outflow (Yamashita et al. 1989) is shown in Figure 1, and a review of other similar cases is given by Rodriguez (1988). Under the interstellar toroid hypothesis the bipolar collimation is a result of large scale ( $0.1 \mathrm{pc}$ ) structures whose density gradients channel the expansion of the gas. Despite a number of problems that are discussed by Rodríguez (1988), it remains an observational fact that most well-studied bipolar outflows show evidence for the presence of an interstellar toroid.

The interstellar toroids are relatively large, self-gravitating structures that appear to be in approximate virial equilibrium. This implies that the ram pressure of the wind should be similar to the gas pressure in the toroid. This can be found to be a reasonable conclusion. Assume for wind parameters a mass loss rate of $3 \times 10^{-7} M_{\odot} \mathrm{yr}^{-1}$ with a velocity of $200 \mathrm{~km} \mathrm{~s}^{-1}$. At a distance of $0.1 \mathrm{pc}$ the wind will have a ram pressure of $3 \times 10^{-10} \mathrm{~g} \mathrm{~cm}^{-1} \mathrm{~s}^{-2}$. This ram pressure is similar to that expected for the gas pressure in the toroid, with typical molecular hydrogen densities 


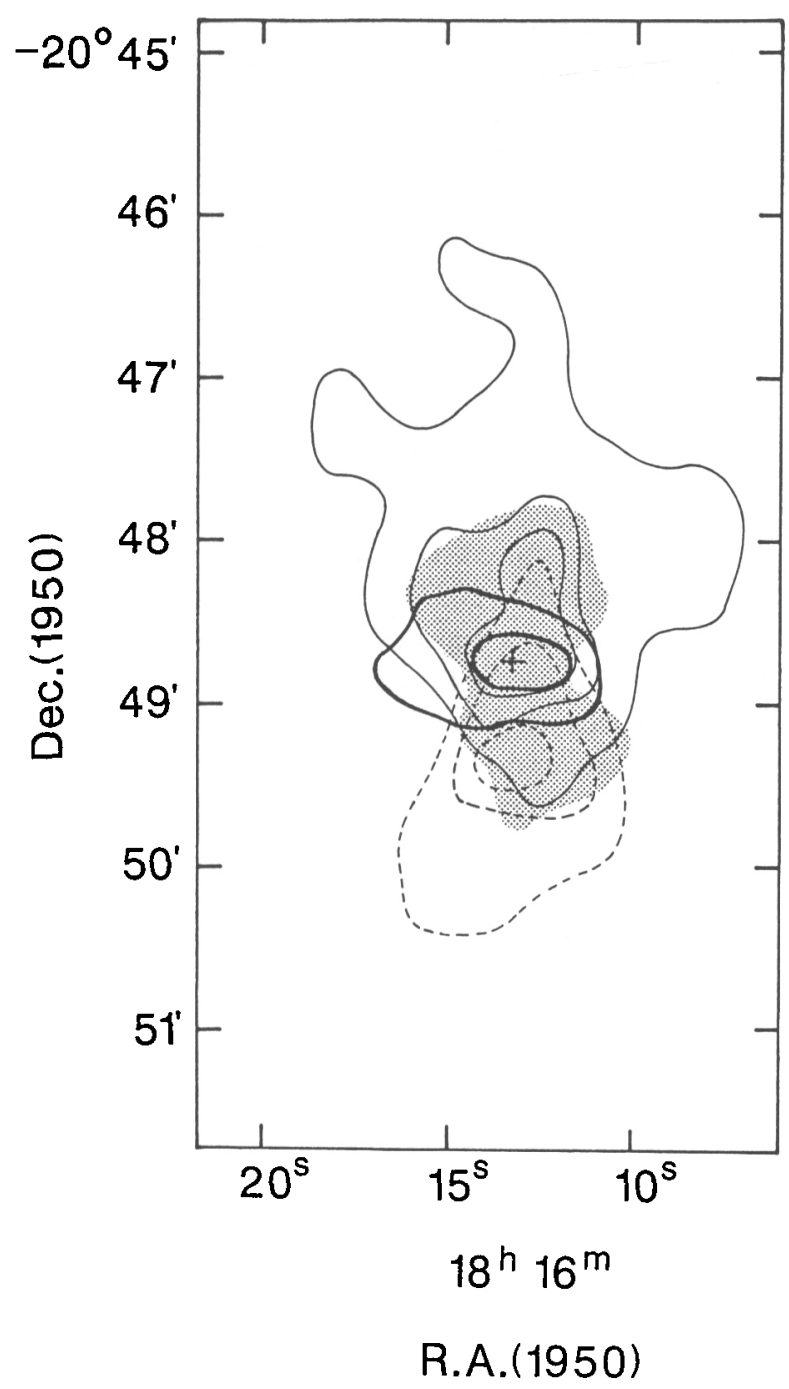

Figure 1. A schematic diagram of the Nobeyama map of the blueshifted (solid line) and redshifted (dashed line) CO $\mathrm{J}=1-0$ bipolar emission associated with the source GGD27 IRS (marked with a cross in the figure). In this figure it is also shown, with thick solid contours, the CS J=2-1 emission around GGD27 IRS. Note the nearly perpendicular alignment between the axis of the bipolar outflow (traced by the $\mathrm{CO}$ ) and the major axis of the dense gas (traced by the CS). The shaded area outlines the extent of the infrared reflection nebula in the region. Data from Yamashita et al. (1989).

of $\sim 10^{4} \mathrm{~cm}^{-3}$ and turbulent velocities of $\sim 1 \mathrm{~km} \mathrm{~s}^{-1}$. In some cases, however, one finds evidence for small, expansive motions (of order of one $\mathrm{km} \mathrm{s}^{-1}$ ) induced by the stellar winds that are powering the bipolar outflows (Torrelles et al. 1987; Marcaide et al. 1988). 


\section{EVIDENCE FOR SMALLER STRUCTURES \\ COLLIMATING THE OUTFLOWS}

During the last years it has become evident that, in addition to any possible large scale collimating mechanisms, there is collimation taking place on scales much smaller than $0.1 \mathrm{pc}$. Rodríguez (1988) has reviewed the direct (images of structures obtained with arc second resolution that resemble circumstellar disks or that can be interpreted as being created with the help of a circumstellar disk) and indirect (usually observations of lower angular resolution that can be interpreted as requiring a disk) evidences for circumstellar disks. Among the direct evidences one can list the optical and radio jets that appear to emanate from some young stars, the infrared halos detected with speckle observations or with maximum entropy reconstructions, and the disklike structures detected with millimeter interferometry.

The best example of a circumstellar disk around a young star is that found around HL Tau by Sargent and Beckwith (1987). The HL Tau disk was detected in the $\mathrm{J}=1-0$ transition of ${ }^{13} \mathrm{CO}$ and has a mass of $\sim 0.1 M_{\odot}$ and a major axis of about $4000 \mathrm{AU}$ (that is, a radius of about $0.01 \mathrm{pc}$, a tenth of that of the typical interstellar toroids). What makes this structure unique is that its kinematics are consistent with Keplerian rotation around a $0.5 M_{\odot}$ central mass. In contrast with the self-gravitating character of the interstellar toroids, the HL Tau disk appears to be gravitationally bound to its star. Then, the adjective circumstellar seems well justified for this object. The high velocity CO outflow found in association with HL Tau (Calvet, Cantó, and Rodríguez 1983) can be interpreted as a bipolar outflow with its axis perpendicular to the major axis of the circumstellar disk (Sato et al. 1989).

Another possible circumstellar disk has been mapped around L1551 IRS 5 by Sargent et al. (1988). This structure is shown in Figure 2. The observations are consistent with the gas being gravitationally bound to the star and its overall properties are fairly similar to those of the HL Tau system.

With the existence of collimation on a small scale one may wonder if larger scale collimation is even needed. An extreme view can be summarized as follows. Assume that the dense core from which the star formed was originally spherical. A star forms at its center and a bipolar wind (collimated by an unspecified mechanism at a scale much smaller than that of the core) starts blowing. This bipolar wind accelerates gas in the core, producing a bipolar outflow. A bipolar cavity will then be cleared, and the remaining dense gas will resemble an interstellar toroid. In this scheme the interstellar toroids are not the cause of the bipolar outflows, but one of the results. Three considerations argue against this point of view. First, for this scheme to work one would require to clear with the bipolar outflow an amount of mass comparable with that present now in the core. Even when a detailed study of this topic has not been undertaken, in general the mass in the high-velocity gas is about one to two orders of magnitude less than the mass in the interstellar toroid. Second, even before star formation takes place, Benson and Myers (1989) have found that dense cores exhibit flattening. This result implies that the future star will find an anisotropic gaseous environment and that its wind will escape more easily in 


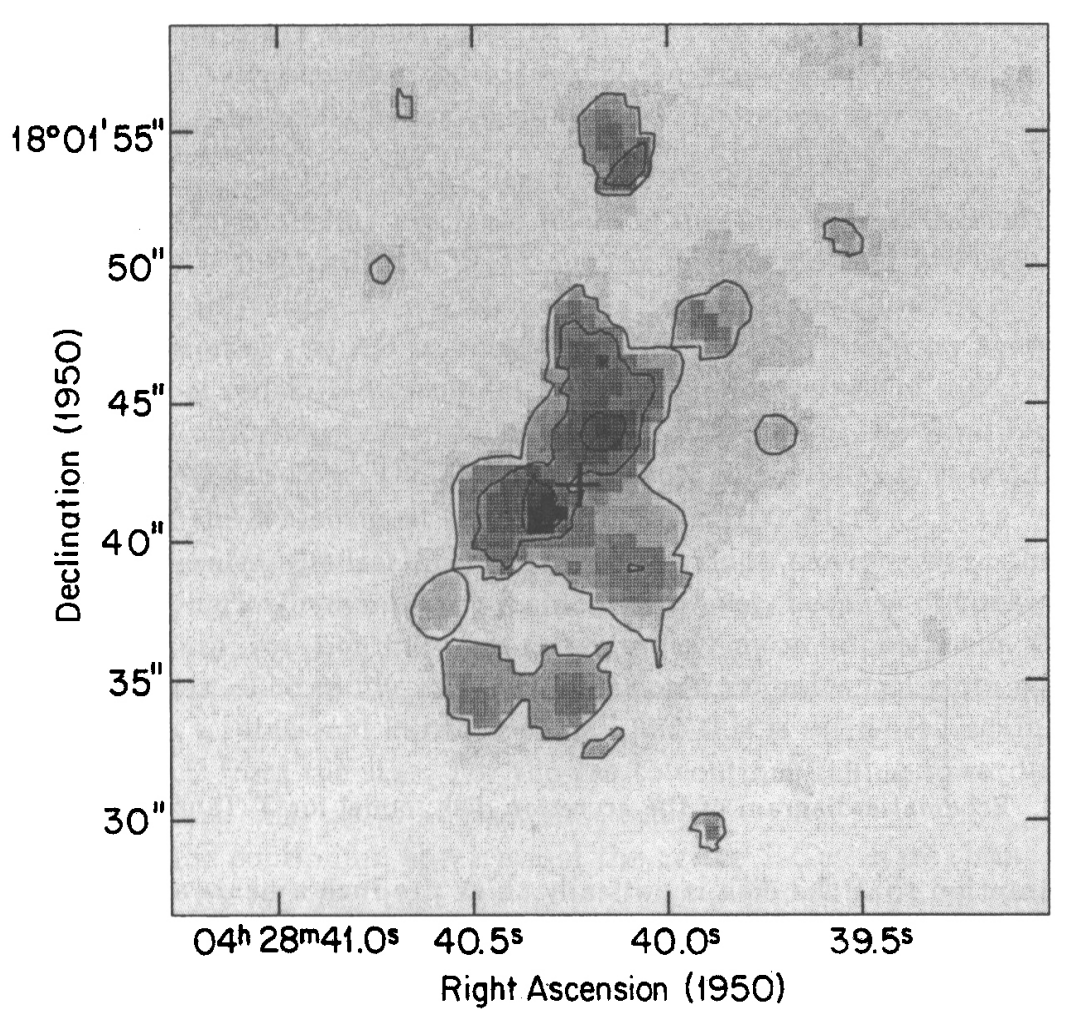

Figure 2. Gray-scale, aperture synthesis map of the integrated $\mathrm{C}^{18} \mathrm{O}$ emission from L1551 IRS 5 at 3.5 arc sec resolution taken from Sargent et al. (1988). The cross marks the position of the $2 \mathrm{~cm}$ compact continuum sources.

the poles of the configuration. Finally, the numerical models of Boss (1987) for the collapse of rotating, axisymmetric, isothermal clouds show that these objects form a protostellar core surrounded by a flattened structure with a central evacuated region, that is, an interstellar toroid.

\section{THEORETICAL WORK ON CIRCUMSTELLAR DISKS}

During the last few years there has been also considerable advancement in the theoretical modeling of circumstellar disks. I will present now a brief summary of the main results of this work, following the papers of Adams and Shu (1986) and Bertout, Basri, and Bouvier (1988). A thin accretion disk in Keplerian rotation around a star is assumed. If the disk's luminosity comes mainly from reprocessing of stellar photons the disk is considered to be passive. An optically thick, but physically thin passive disk will have a luminosity of about $1 / 4$ of that of the star. If viscous dissipation in the accretion disk results in significant heating and production of intrinsic luminosity, the disk is considered to be active. The luminosity of an active disk can equal and even exceed that of the star. In both passive and active disks the temperature of the disk depends with distance to the star as $T_{D} \propto r^{-3 / 4}$. This temperature dependence 


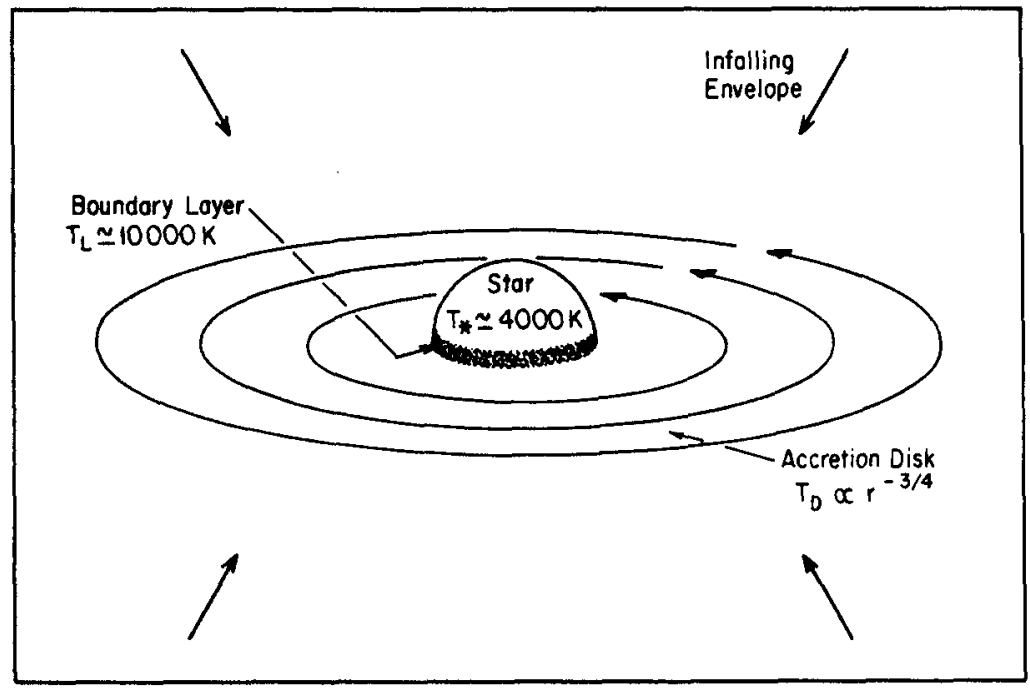

Figure 3. Schematic diagram of the accretion disk model for T Tauri stars.

and the assumption that the disk is optically thick produce a near- and middleinfrared spectrum with a flux density depending on frequency as $S_{\nu} \propto \nu^{1 / 3}$. Even when some sources do show this spectral index, the most extreme T Tauri stars (that is, those with larger infrared excesses, ultraviolet excesses, variability, and line emission) have a flat near- and middle-infrared spectrum. To alleviate this problem, it has been proposed that the outer parts of the disk must radiate comparatively more that in the orthodox model (Kenyon and Hartmann 1987; Adams, Lada, and Shu 1988).

One additional ingredient in these disk models is the presence of a boundary layer. The gas being accreted will dissipate one half of its gravitational energy by viscous processes in the disk. The other half will be dissipated as the rapidly rotating ( $\mathrm{a}$ few hundred $\mathrm{km} \mathrm{s}^{-1}$ ) gas in the inner part of the disk is abruptly incorporated to the slowly rotating ( $\mathrm{a}$ few tens of $\mathrm{km} \mathrm{s}^{-1}$ ) equator of the star. The characteristics of the boundary layer where this transformation of kinetic energy into heat and radiation takes place are poorly known, but it is believed that temperatures as high as $10^{4} \mathrm{~K}$ could be obtained. This relatively hot region could account for the ultraviolet excesses known to exist in some $\mathrm{T}$ Tau stars. Finally, an infalling envelope surrounds the star and disk. A schematic depiction of the components of the accretion disk model for $\mathrm{T}$ Tauri stars is shown in Figure 3.

Bertout, Basri, and Bouvier (1988) have discussed that the spectral shape of the continuum of $T$ Tau stars, from the ultraviolet to the infrared, can be explained in a general sense if we assume that the stars are chromospherically active and that they have an accretion disk. Reasonable accretion rates of $\sim 10^{-7} \mathrm{M}_{\odot} \mathrm{yr}^{-1}$ are required. This remarkable achievement leads one to try to use this model as a paradigm that 
could explain other observations. As a radio observer, I would like to emphasize some radio results that are not being considered by the present accretion disk models and that could perhaps be explained with additional theoretical work.

\section{IV a. The Size of the Disk}

The theoretical modeling available refers in most cases to disks with a radius of about $100 \mathrm{AU}$. It is interesting to point out the arguments that are usually given by the theoreticians to justify their adoption of a $100 \mathrm{AU}$ radius for the disk.

1) A dimension of $100 \mathrm{AU}$ is, roughly, the size of our planetary system.

2) The lack of redshifted forbidden line emission observed in many $T$ Tau stars suggests that an absorbing screen hides the receding part of the wind from our view (Edwards et al. 1987). From the emission measure of the lines it is estimated that the minimum size of the screen must be on the order of $100 \mathrm{AU}$.

3) At large scales, the infalling gas in the envelope moves toward the disk in a more or less spherically symmetric fashion. However, because of conservation of angular momentum, rotation becomes progressively more important in the kinematics of the infalling gas. The radius at which rotation dominates the motion of gas can be considered as a rotational barrier to infall and this is estimated again to happen at around $100 \mathrm{AU}$ from the star. For the gas to continue falling toward the star an accreting disk must exist up to at least that radii.

4) Finally, the continuum spectrum of the active $T$ Tau stars shows a bend at around $3 \times 10^{13} \mathrm{~Hz}(10 \mu \mathrm{m})$ that can be modeled if the disk has an outer radius of about 100 AU (Adams, Lada, and Shu 1988).

Even when the $100 \mathrm{AU}$ radius may actually turn out to be the typical value for accretion disks, it is important to note that the observational results of Sargent and collaborators indicate the presence of structures about an order of magnitude larger. Even when the object is at a different evolutionary stage, the famous dust disk around $\beta$ Pictoris has a radius of about $400 \mathrm{AU}$ (Smith and Terrile 1985), again significantly larger than $100 \mathrm{AU}$. I consider very important to understand the relation between the inner $100 \mathrm{AU}$, that have been modeled in great detail, and the larger structures found observationally.

\section{IV b. The Collimation of the Outflows}

While the interstellar size structures have been modeled in considerable detail to explain how they could collimate the observed bipolar outflows (see, for example, the papers of Barral and Cantó 1981; Königl 1982; and Pudritz and Norman 1983), very little theoretical work has been made with circumstellar accretion disks in what refers to their role as collimators of bipolar outflows and jets. Shu, Adams, and Lizano (1987) note that, gradually, the gas infalling from the envelope will fall preferentially onto the disk rather than onto the star. The stellar wind will then break out along the rotational axis of the system (the channels of weakest resistance), creating a bipolar outflow. A detailed modeling of this scenario would be most valuable.

\section{IV c. The Radio Continuum Excess}

The models for the continuum emission from accretion disks can be extrapolated to the radio wavelengths, where they predict a negligible flux. It is known, however, that $\mathrm{T}$ Tau stars are frequently associated with radio continuum emission at levels that are orders of magnitude larger than expected from dust emission in the disk. 
Furthermore, high angular resolution Very Large Array observations of some objects indicate that the radio emission is coming from within $100 \mathrm{AU}$ from the star (Bieging and Cohen 1985; Rodríguez et al. 1986; Rodríguez et al. 1989), the same volume that is supposed to contain the accretion disk. This radio continuum excess probably is free-free emission arising from shock-ionized gas produced as the stellar wind interacts with the infalling gas. But again, successful modeling has not been achieved.

\section{RADIO CONTINUUM FROM HERBIG-HARO OBJECTS AND THEIR EXCITING SOURCES}

The Herbig-Haro objects are optical nebulosities excited by a shock wave produced by the collimated wind of a young star. Since they are formed by ionized gas it is expected that they will emit free-free radiation in the radio wavelenghts at some level. Herbig-Haro 1 and 2 were detected with the VLA by Pravdo et al. (1985) at the mJy level. These authors also detected the central exciting source of the system, VLA 1 , a heavily obscured pre-main-sequence object with a luminosity of $\sim 50 L_{\odot}$ (Harvey et al. 1986). The detailed VLA study of the HH1-2 region by Rodríguez et al. (1989) has produced a significant advance in our understanding of the HH phenomenon. When observed with sub arc sec angular resolution, VLA 1 appears elongated and its major axis is aligned within a few degrees with the HH12 axis. The dependences with frequency of the flux density and of the angular size of the major axis of VLA 1 can be explained in terms of a confined, thermal jet of bipolar geometry. The radio emission from HH1 and 2 is optically-thin free-free radiation and it has been possible to confirm their proper motions, first observed in the optical by Herbig and Jones (1981), comparing VLA data taken only two years apart.

Searches for radio continuum emission from other classic HH objects proved unsuccessful (Lane 1989; Curiel et al. 1989) most probably because HH1 and 2 are the more luminous objects of the class. However, as I discussed in the Introduction, the $\mathrm{HH}$ phenomenon is not restricted to low luminosity stars and one could expect the $\mathrm{HH}$ objects associated with more luminous stars to be intrinsically more luminous and thus easier to detect in the radio. This expectation has proved to be correct and during this year there have been reports of radio continuum emission from several $\mathrm{HH}$ objects associated with massive stars. Along these lines of research, HH80-81 (Rodríguez and Reipurth 1989), Cep A West (=the HW object; Hughes 1989), M42/HH3-4 (Yusef-Zadeh 1989), and M16/HH1 (Curiel et al. 1990) have been detected. Figure 4 shows a VLA image of the Orion region that shows, to the southeast of the Orion bar, radio continuum emission from M42/HH3-4 (Yusef-Zadeh 1989).

One problem in the study of these HH objects is that in some cases their nature as bona fide $\mathrm{HH}$ objects is uncertain. In particular, in some of these objects the radiative contribution to the excitation of the $\mathrm{HH}$ object could be important. It is perhaps because of this problem that the study of suspected HH objects associated with massive stars did not receive much attention. However, the HH nature is well established at least in the cases of HH80-81 (Reipurth and Graham 1988; Heathcote and Reipurth 1989) and Cep A West (Hartigan, Raymond, and Hartmann 1987). The objects $\mathrm{HH} 80-81$ are intrinsically 10 times more luminous in the radio than $\mathrm{HH} 1-2$, 


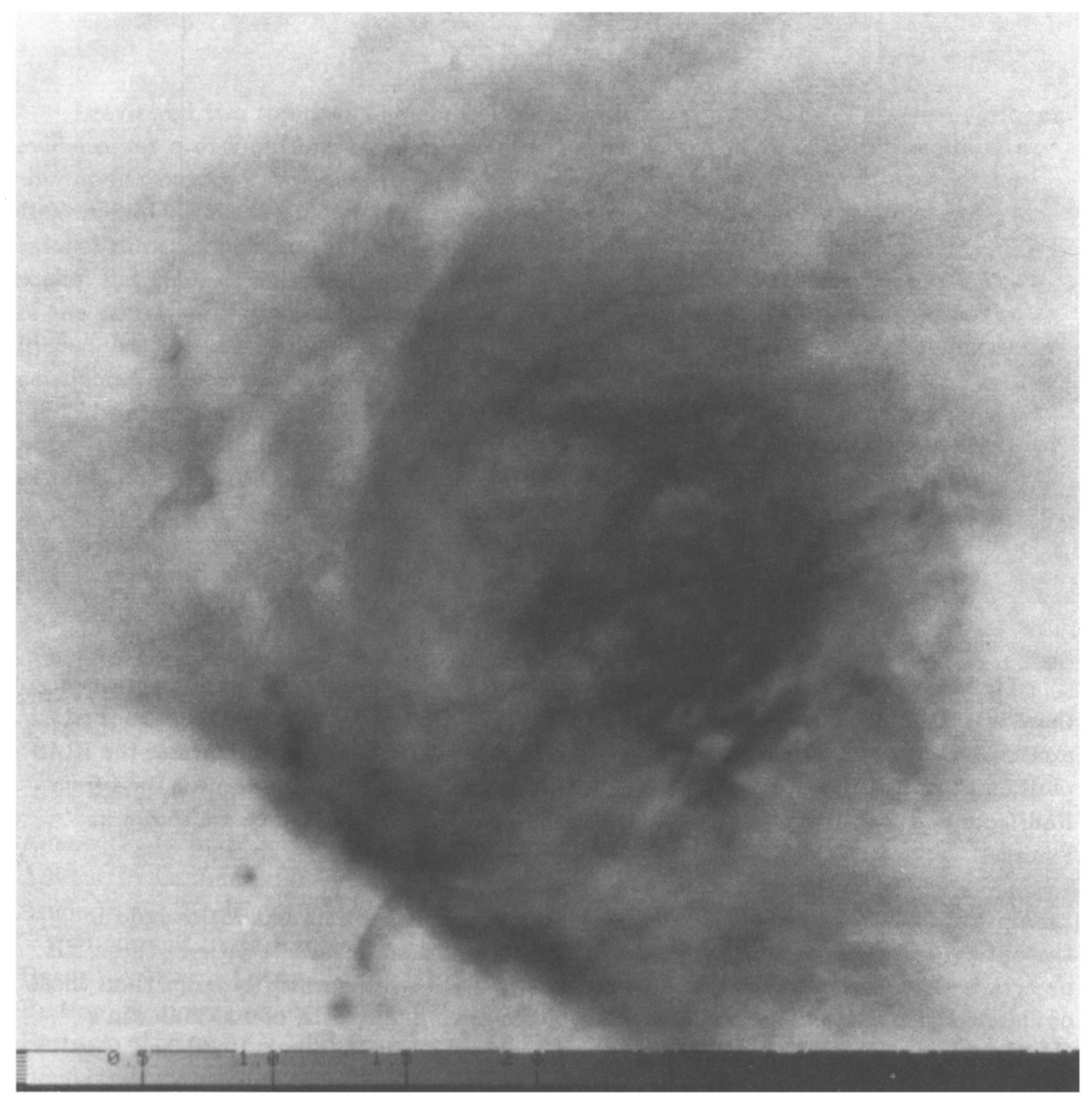

Figure 4. VLA image of the Orion region made at $6 \mathrm{~cm}$. The Orion nebula occupies most of the figure. M42/HH3-4 (Walsh 1982) are the faint nebulosities to the southeast of the Orion bar (bottom left corner of the image). Data from Yusef-Zadeh (1989).

while Cep A West is intrinsically 30 times more luminous in the radio than HH1-2. Since the radio emission is expected to be correlated, to first approximation, with 


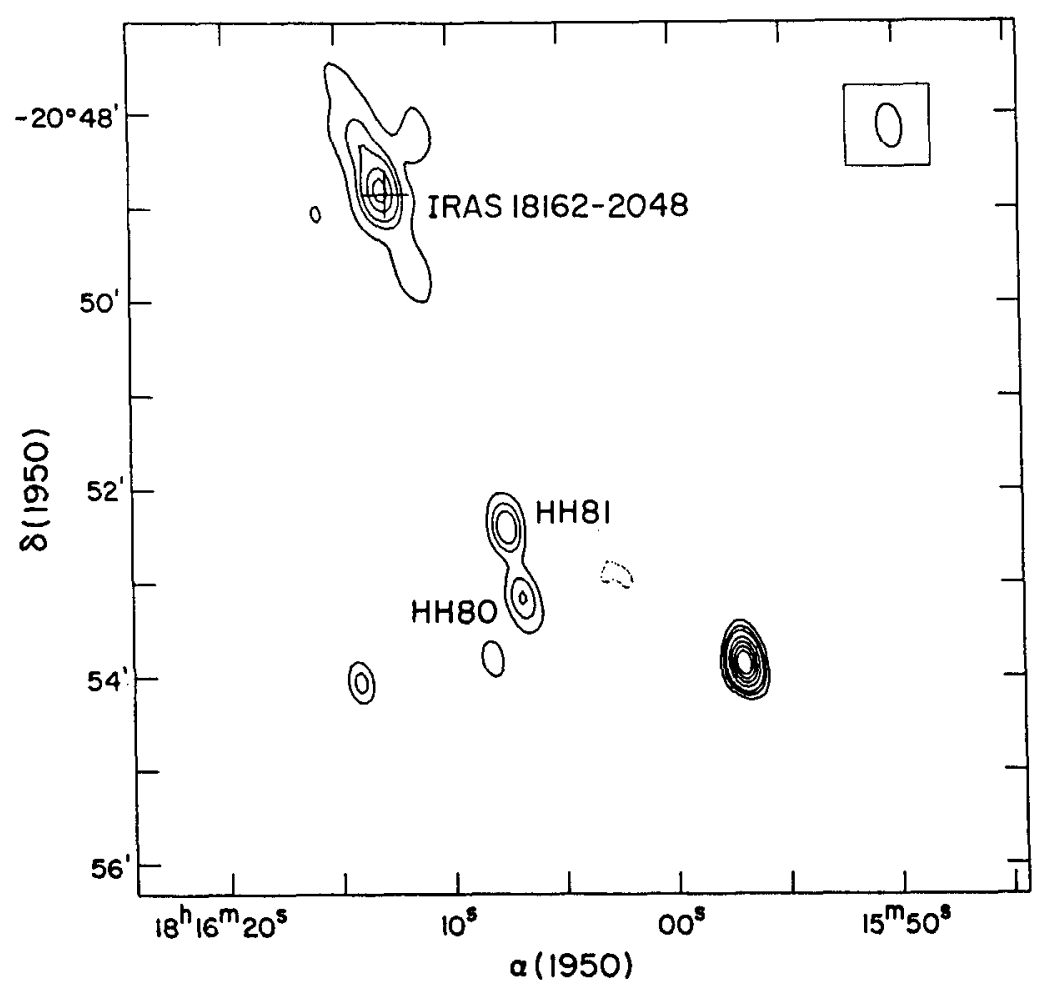

Figure 5. VLA map of the 6-cm emission from the HH80-81 region. The positions of these two HH objects, as well as that of IRAS 18162-2048 (=GGD27 IRS), the suspected exciting source of the region, are indicated in the figure. The large cross indicates the IRAS position. The half power contour of the beam is shown in the top right corner. Data from Rodriguez and Reipurth (1989). Note that additional data on this source are shown in Figure 1.

the optical emission (Pravdo et al. 1985), it is probable that similar ratios hold in the optical (after correction for extinction). An additional characteristic of these $\mathrm{HH}$ objects is that they possess optical line emission with widths much broader than those of classic $\mathrm{HH}$ objects. While classic $\mathrm{HH}$ objects have line widths of $100-200 \mathrm{~km} \mathrm{~s}^{-1}$, HH80-81 have line widths of $700 \mathrm{~km} \mathrm{~s}^{-1}$ (Heathcote and Reipurth 1989) and Cep A West has line widths of $500 \mathrm{~km} \mathrm{~s}^{-1}$ (Hartigan, Raymond and Hartmann 1987). This result is quite important because it may imply that the winds powering these $\mathrm{HH}$ objects have higher velocities than those powering classic $\mathrm{HH}$ objects, as expected since the former are associated with more massive stars than the latter.

The HH objects 80 and 81 have been studied in some detail recently (Reipurth and Graham 1988; Rodriguez and Reipurth 1989). These $\mathrm{HH}$ objects are being excited by a $10^{4} L_{\odot}$ infrared source, GGD27 IRS (=IRAS 18162-2048), the same object presented in Figure 1. This exciting source is also detectable in the radio continuum (Figure 5), and exhibits elongated morphology with its major axis pointing 
approximately to HH80-81. The projected separation between IRAS 18162-2048 and HH80-81 is $\sim 2 \mathrm{pc}$, while that between VLA 1 and $\mathrm{HH} 1$ or HH2 is $\sim 0.2 \mathrm{pc}$. The HH80-81 system may be, in some sense, a scaled-up version of HH1-2. Of course, HH1 and 2 are located symmetrically with respect to VLA 1 and to consolidate the analogy one would expect to find HH objects to the northeast of IRAS 18162-2048.

\section{CONCLUSIONS}

I reviewed two topics in the area of outflows from young stars. The observational evidence for a disk-outflow connection (in the sense that the disks help to collimate the bipolar outflows) appears to be considerable, in particular for structures in the $1000 \mathrm{AU}$ to $0.1 \mathrm{pc}$ size range. It is possible that circumstellar accretion disks of even smaller dimensions could be collimating the outflows at the $100 \mathrm{AU}$ and smaller scales, but there is not yet a quantitative model available. Given the relative success of the accretion disk models in explaining the continuum of $T$ Tauri stars from the ultraviolet to the infrared, I have pointed out several radio results that have not been considered by these models.

The second and last part of this review discusses recent radio continuum observations of Herbig-Haro objects and their exciting sources. In particular, the field of radio observations of $\mathrm{HH}$ objects associated with massive stars has grown rapidly in the last couple of years. These $\mathrm{HH}$ objects are intrinsically much more luminous in the radio and possess larger line widths in the optical than the classic HH objects.

\section{ACKNOWLEDGEMENTS}

I gratefully acknowledge support from a Guggenheim Fellowship. I am also thankful to F. C. Adams, J. Cantó, and P. Hartigan for their valuable comments. F. Yusef-Zadeh kindly provided the VLA image shown in Figure 4.

\section{REFERENCES}

Adams, F. C. and Shu, F. H. 1986, Ap. J., 308, 836.

Adams, F. C., Lada, C. J., and Shu, F. H. 1988, Ap. J., 326, 865.

Anglada, G., Rodríguez, L. F., Torrelles, J. M., Estalella, R., Ho, P. T. P., Cantó, J., López, R., and Verdes-Montenegro, L. 1989, Ap. J., 341, 208.

Barfal, J. F. and Cantó, J. 1981, Rev. Mexicana Astron. Astrof., 5, 101.

Benson, P. J. and Myers, P. C. 1989, Ap. J. Suppl., 000, 000.

Bertout, C. Basri, G., and Bouvier, J. 1988, Ap. J., 330, 350.

Bieging, J. H. and Cohen, M. 1985, Ap. J. (Letters), 289, L5.

Boss, A. P. 1987, Ap. J., 316, 721.

Calvet, N., Cantó, J., and Rodríguez, L. F. 1983, 268, 739.

Cantó, J., Goudis, C., Johnson, P. G., and Meaburn, J. 1980, Astr. Ap., 85, 128.

Curiel, S., Rodríguez, L. F., Cantó, J., and Torrelles, J. M. 1989, Rev. Mexicana Astron. Astrof., 17, 000.

Curiel, S. et al. 1990, in preparation.

Edwards, S., Cabrit, S. Strom, S. E., Heyer, I., Strom, K. M., and Henderson, E. 1987, Ap. J., $321,473$. 
Gull, T. R., Goad, L., Chiu, H. Y., Maran, S. P., and Hobbs, R. W. 1973, Publ. A. S. P., 85, 526.

Gyulbudaghian, A. L., Glushkov, Yu. I., and Denisyuk, E. K. 1978 Ap. J. (Letters), 224, L137.

Hartigan, P., Raymond, J. and Hartmann, L. 1987, Ap. J., 316, 323.

Heathcote S. and Reipurth, B. 1989, in preparation.

Herbig, G. H. and Jones B. F. 1981, A. J., 86, 1232.

Hughes, V. A. 1989, A. J., 97, 1114.

Kenyon, S. J. and Hartmann, L. 1987, Ap. J., 323, 714.

Königl, A. 1982, Ap. J., 261, 115.

Lane, A. P. 1989 , in preparation.

Marcaide, J. M., Torrelles, J., Güsten, R., Menten, K., Ho, P. T. P., Moran, J. M., and Rodríguez, L. F. 1988, Astr. Ap., 197, 235.

Meaburn, J. and White, N. J. 1982, M. N. R. A. S., 199, 121.

Münch, G. 1977, Ap. J. (Letters), 212, L77.

Myers, P. C. 1985, in Protostars and Planets II, ed. D. C. Black and M. S. Matthews (University of Arizona), p. 81.

Myers, P. C., Heyer, M., Snell, R. L., and Goldsmith, P. F. 1988, Ap. J., 324, 907.

Pravdo, S. H., Rodríguez, L. F., Curiel, S., Cantó, J., Torrelles, J. M., Becker, R. H., and Sellgren, K. M. 1985, Ap. J. (Letters), 293, L35.

Pudritz, R. E. and Norman, C. A. 1983, Ap. J., 274, 677.

Reipurth, B. and Graham, J. A. 1988, Astr. Ap., 202, 219.

Rodríguez, L. F., Cantó, J., Torrelles, J. M., and Ho, P. T. P. 1986, Ap. J. (Letters), 301, L25.

Rodríguez, L. F. 1988, in Galactic and Extragalactic Star Formation, ed. R. E. Pudritz and M. Fich (Kluwer Academic Publishers), p. 97

Rodríguez, L. F., Ho, P. T. P., Torrelles, J. M., Curiel, S. and Cantó, J. 1989, submitted to Ap. J.

Rodríguez, L. F. and Reipurth, B. 1989, Rev. Mexicana Astron. Astrof., 17, 59.

Sargent, A. I. and Beckwith S. 1987, Ap. J., 323, 294.

Sargent, A. I., Beckwith S., Keene, J., and Masson, C. 1988, Ap. J., 333, 936.

Sato, S. et al.. 1989, preprint.

Shu, F. H., Adams, F. C., and Lizano, S. 1987, Ann. Rev. Astr. Ap., $25,23$.

Smith, B. A. and Terrile, R. J. 1985, Science, 226, 1421.

Strom, K. M., Strom, S. E., Wolff, S. C., Morgan, J., and Wenz, M. 1986, Ap. J. Suppl., $62,39$.

Torrelles, J. M., Rodríguez, L. F., Cantó, J., Carral, P., Marcaide, J., Moran, J. M., and Ho, P. T. P. 1983, Ap. J., 274, 214.

Torrelles, J. M., Ho, P. T. P., Moran, J. M., Rodríguez, L. F., and Cantó, J. 1986, Ap. J., 307, 787.

Torrelles, J. M., Ho, P. T. P., Rodríguez, L. F., Cantó, J., and Moran, J. M. 1987, Ap. J., 321, 884.

Walsh, J. R. 1982, M. N. R. A. S., $201,561$.

Yamashita, T., Suzuki, H., Kaifu, N., Tamura, M., Mountain, C. M., and Moore, T. J. T. 1989, submitted to Ap. J.

Yusef-Zadeh, F. 1989, in preparation. 


\section{Discussion:}

DYSON: Is it clear whether your "super" HH objects are shock or radiatively excited?.

RODRIGUEZ: Not in all, and this problem has certainly impeded the advancement in the understanding of this interesting variety of $\mathrm{HH}$ objects. However, in the case of HH8081, the studies of Reipurth and collaborators strongly support a shock-excited nature.

MÜNCH: In relation to the comment made by J. Dyson I would say that some of the condensations seen near $\theta^{2}$ Ori, south of the Bar, are not quite alike the HH-objects seen in low temperature regions. One of them has a central star, of G-K type, a roundish appearence and with an extension in directions opposite to that of $\theta^{2}$ Ori. It looks as if the UV-radiation of $\theta^{2}$ has substantially altered the surrounding medium against which the objects is expanding at low velocity. The object near the BN and Low-Kleinman nebula, on the other hand, is a true HH-object, with obvious signatures of shock $\left(240 \mathrm{kms}^{-1}\right)$ in its spectrum. The question I would like to ask to Luis Felipe is whether in the molecular disks any indications of anomalous isotopic composition have been found. In our planetary system isotopic anomalies have been found and are explained by hard particle radiation (soft cosmic rays) that may have been emitted by the Sun in its $T$ Tau phase.

RODRIGUEZ: We are only starting to study these disks. The isotopic anomalies may be present only very close to the star, involving relatively little gas. I belive that in the future, with increased sensivity, your question could be addressed.

HUGHES: In addition to the HH objects, for which a model is that of an expanding shock, there appears to be HH-objects produced by bullets. For example, GGD-37 appears to contain bullets with speeds of up to $500 \mathrm{kms}^{-1}$. Do you have any comment on this, and are there 2 types of $\mathrm{HH}$ objects?

RODRIGUEZ: You are correct in pointing out GGD37 as an HH object powered by a massive star. As is discussed in the review by Cantó, the HH phenomenon can appear at least in four different scenarios.

NATTA: You said that probably the momentum in the wind is much lower than the momentum in the $\mathrm{CO}$ outflow. I think that most of the wind is in the form of neutral gas, and that indeed the two may be very close.

RODRIGUEZ: I agree with you that the winds could be largely neutral. In the case of HH7-11 the results of Lizano et al. (1988) support this view. 